\section{Was hilft wirklich bei Fatigue?}

Krebspatienten, die unter Fatigue-Symptomen leiden, versucht man mit Sport, Psychotherapie oder Medikamenten wieder zu mehr Leistungsfähigkeit zu verhelfen. Von welcher Intervention profitieren die Betroffenen am meisten?

E ast alle Krebspatienten fühlen sich während oder nach der Behandlung dauerhaft schlapp, ausgelaugt und müde. Manchmal verschwindet das Erschöpfungssyndrom von selbst, bei vielen halten die Beschwerden über Monate oder Jahre an. Leiden Krebspatienten dauerhaft, wird üblicherweise mit Sport, Psychotherapie oder Medikamenten versucht gegenzusteuern. Doch offenbar sind nicht alle Behandlungsansätze gleich wirksam, wie sich in einer USamerikanischen Metaanalyse kürzlich herausstellte. Die Onkologin Karen Mustian vom Wilmot Cancer Institute in Rochester und Kollegen anderer US-amerikanischer Krebszentren haben in einer Metaanalyse die Wirksamkeit der vier gängigsten Behandlungskonzepte überprüft. Dazu gehören neben Bewegung und Psychotherapie, auch die Kombination aus beidem sowie die medikamentöse Behandlung mit Paroxetin, Modafinil bzw. Armodafinil, Methylphenidat bzw. Dexmethylphenidat, Dexamphetamin oder Methylprednisolon.

Die Onkologen berücksichtigten nur randomisiert kontrollierte Studien mit erwachsenen Krebspatienten, die explizit die Intensität der tumorbedingten Fatigue als Studienziel definiert hatten und diese nicht nur als Nebenwirkung gängiger Krebstherapien berücksichtigten. Der Einsatz von Erythropoetin war ebenso ein Ausschlusskriterium wie alternativ- oder komplementärmedizinische Therapien. Schließlich genügten 113 Studien den Auswahlkriterien und wurden in die Analyse einbezogen.

Den größten Nutzen zogen Krebspatienten, die mit Sport versuchten, die Fatigue zu lindern. Auch mit einer Psychotherapie oder der Kombination aus Sport und Psychotherapie ließen sich gute Erfolge erzielen. Mit Medikamenten hingegen konnte den Patienten kaum aus dem körperlichen und emotionalen Tief geholfen werden. Gemäß den Ergebnissen der Metaanalyse kann die Wirksamkeit in Abhängigkeit verschiedener Begleit- faktoren variieren: Neben dem Tumorstadium ist der Zeitpunkt entscheidend, zu dem mit der Intervention begonnen wird . Demnach war der größte Benefit bei Tumoren im Frühstadium sowie abgeschlossener Krebstherapie zu verzeichnen. Gruppen- oder Einzeltherapie beeinflussen die Wirksamkeit ebenso wie ein regelmäßiger Kontakt zum Therapeuten, die Psychotherapieform oder das Behandlungskonzept, das in den Kontrollgruppen zum Einsatz kommt. Studien mit einer Intent-to-treat-Analyse sowie Studien, in denen die Fatigue-Intensität anhand der Piper-Fatigue-Skala gemessen wurde, wiesen einen im Durchschnitt größeren Nutzen aus.
Mit Sport, Psychotherapie oder einer Kombination aus beidem lassen sich die Symptome eines tumorbedingten Erschöpfungssyndroms während und nach der Krebstherapie hervorragend verbessern, so das Resümee der Onkologen. Von Medikamenten hingegen profitierten die Patienten kaum. Die Metaanalyse zeige allerdings auch, dass sich die Wirksamkeit von Sport und Psychotherapie mit Fortschreiten der Krebstherapie verändert. So profitieren Patienten, die sich beispielsweise mitten in der Krebstherapie befinden, von körperlicher Bewegung in besonderem Maße. Ist die primäre Therapie hingegen abgeschlossen, ist dem Patienten mit Psychotherapie oder Psychotherapie kombiniert mit körperlicher Bewegung am besten zu helfen.

$(d k)$

Mustian KM et al. Comparison of Pharmaceutical, Psychological, and Exercise Treatments for Cancer-Related Fatigue - A Meta-analysis. JAMA Oncol 2017; online 2. März.

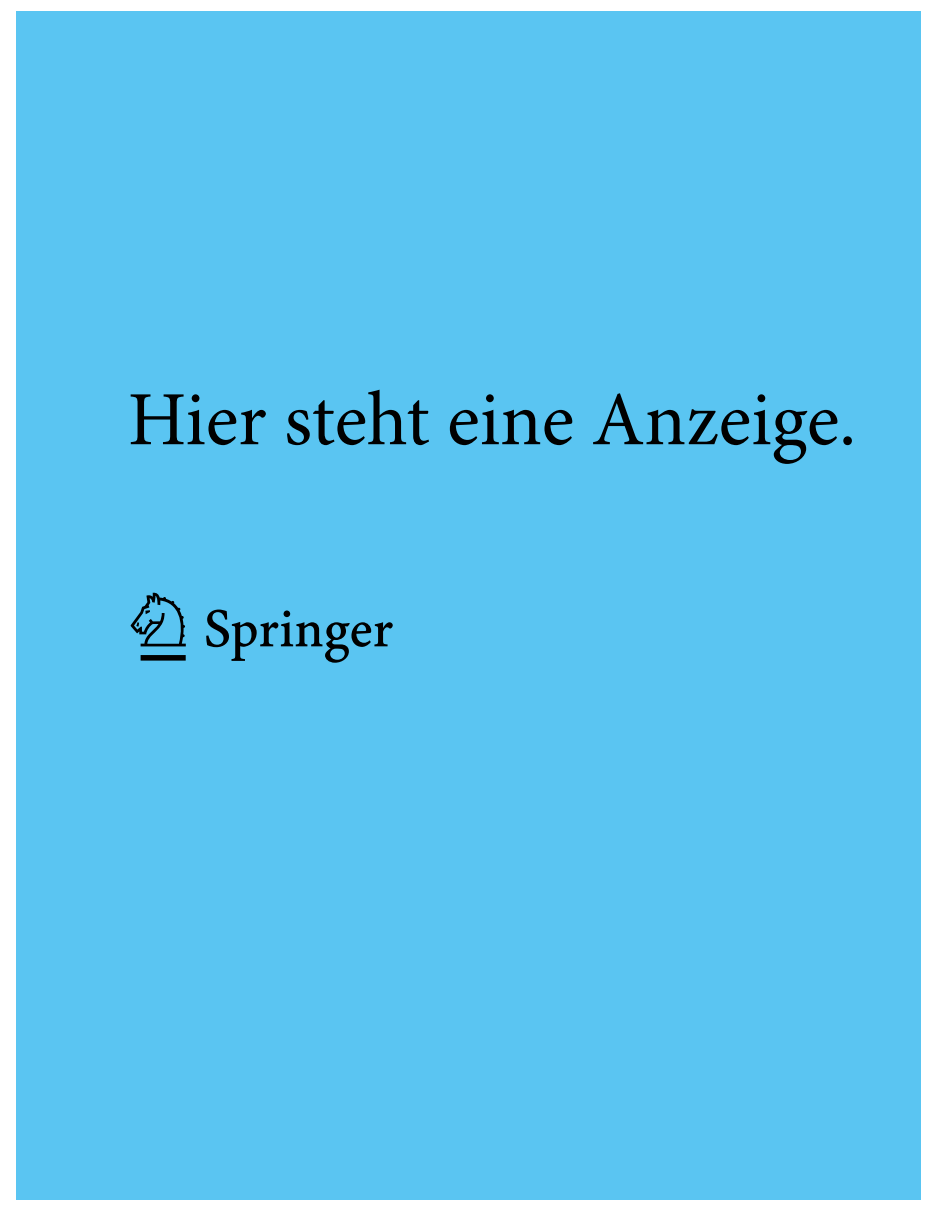

